\title{
Investigation of the role of ACTPR2 in kiwifruit and its response to Botrytis cinerea infection
}

\author{
Zhe-Xin $\mathrm{Li}^{1+}$, Jian-Bin Lan ${ }^{1 \dagger}$, Yi-Qing Liu', Li-Wang Qi ${ }^{2^{*}}$ and Jian-Min Tang ${ }^{1 *}$ (B)
}

\begin{abstract}
Background: Elucidation of the regulatory mechanism of kiwifruit response to gray mold disease caused by Botrytis cinerea can provide the basis for its molecular breeding to impart resistance against this disease. In this study, 'Hongyang' kiwifruit served as the experimental material; the TOPLESS/TOPLESS-RELATED (TPL/TPR) co-repressor gene ACTPR2 was cloned into a PTRV2 vector (ACTPR2-TRV) and the virus-induced gene silencing technique was used to establish the functions of the ACTPR2 gene in kiwifruit resistance to Botrytis cinerea.

Results: Virus-induced silencing of ACTPR2 enhanced the susceptibility of kiwifruit to Botrytis cinerea. Defensive enzymes such as superoxide dismutase (SOD), peroxidase (POD), catalase (CAT), and phenylalanine ammonia-lyase (PAL) and endogenous phytohormones such as indole acetic acid (IAA), gibberellin (GA $)_{3}$, abscisic acid (ABA), and salicylic acid (SA) were detected. Kiwifruit activated these enzymes and endogenous phytohormones in response to pathogen-induced stress and injury. The expression levels of the IAA signaling genes-ACNIT, ACARF1, and ACARF2were higher in the ACTPR2-TRV treatment group than in the control. The IAA levels were higher and the rot phenotype was more severe in ACTPR2-TRV kiwifruits than that in the control. These results suggested that AcTPR2 downregulation promotes expression of IAA and IAA signaling genes and accelerates postharvest kiwifruit senescence. Further, Botrytis cinerea dramatically upregulated ACTPR2, indicating that ACTPR2 augments kiwifruit defense against pathogens by downregulating the IAA and IAA signaling genes.
\end{abstract}

Conclusions: The results of the present study could help clarify the regulatory mechanisms of disease resistance in kiwifruit and furnish genetic resources for molecular breeding of kiwifruit disease resistance.

Keywords: ACTPR2, Botrytis cinerea, IAA signaling, Kiwifruit, Virus-induced gene silencing

\footnotetext{
*Correspondence: Iwai@caf.ac.cn; Tangjmjy@163.com

${ }^{\dagger}$ Zhe-Xin Li and Jian-Bin Lan contributed equally to this work.

${ }^{2}$ State Key Laboratory of Tree Genetics and Breeding, Research Institute of

Forestry, Chinese Academy of Forestry, Beijing 100091, P.R. China

'Chongqing Key Laboratory of Economic Plant Biotechnology, Collaborative

Innovation Center of Special Plant Industry in Chongqing, College of

Landscape Architecture and Life Science/ Institute of Special Plants,

Chongqing University of Arts and Sciences, Yongchuan 402160, P.R. China
}

(C) The Author(s). 2020 Open Access This article is licensed under a Creative Commons Attribution 4.0 International License, which permits use, sharing, adaptation, distribution and reproduction in any medium or format, as long as you give appropriate credit to the original author(s) and the source, provide a link to the Creative Commons licence, and indicate if changes were made. The images or other third party material in this article are included in the article's Creative Commons licence, unless indicated otherwise in a credit line to the material. If material is not included in the article's Creative Commons licence and your intended use is not permitted by statutory regulation or exceeds the permitted use, you will need to obtain permission directly from the copyright holder. To view a copy of this licence, visit http://creativecommons.org/licenses/by/4.0/. The Creative Commons Public Domain Dedication waiver (http://creativecommons.org/publicdomain/zero/1.0/) applies to the data made available in this article, unless otherwise stated in a credit line to the data. 


\section{Background}

Kiwifruit (Actinidia chinensis L.) is prone to fungal pathogen infections that cause major postharvest crop losses and may render the fruit unsafe for consumers. Botrytis cinerea (B. cinerea) is a fungal pathogen responsible for gray mold. It can damage or destroy $\leq 30 \%$ of the kiwifruit crop [1]. In order to breed gray mold resistance into kiwifruit, it is first necessary to elucidate the mechanism regulating plant pathogen response. Current research on gray mold control has focused mainly on physical, chemical, and certain biological controls [2-5]. However, the regulatory and signaling pathways associated with the genes controlling disease resistance in kiwifruit remain to be determined.

Members of the TOPLESS/TOPLESS-RELATED (TPL/TPR) co-repressor protein family interact with transcription factors [6]. The TPL/TPR domains include the highly conserved $\mathrm{N}$-terminal TPD region comprising the lissencephaly homologous (LisH) dimerization motif, a C-terminal to the LisH (CTLH) motif, and C-terminal WD40-repeats [7, 8]. The TOPLESS domains (TPDs) mediate TPL/TPR oligomerization and interact with proteins containing the EAR motif [9]. Most EAR motifs were detected in proteins regulating the transcription of signaling pathways for phytohormones such as auxin, abscisic acid, gibberellins, salicylic acid, ethylene, and jasmonate [10-14].

It was first reported that TPL/TPR co-repressors directly interact with the WUSCHEL (WUS) transcription factor in Arabidopsis thaliana [6]. The TPL/TPR co-repressor family members, TPL and TPR4, interact with WUS. Five TPL/TPR family genes including TPL and TPR1-TPR4 were detected in Arabidopsis [15]. The TPL/TPR co-repressors play vital roles in plant growth and development [10,16-18]. A defective tpl mutation, nonetheless, permits normal embryonic development in Arabidopsis. Hence, TPL protein function is redundant and may be replaced by four other homologous proteins. When tpl/tpr1/tpr3/tpr4 quadruple mutant lines were transformed with TPR2 protein RNAi, abnormal embryonic development occurred. Thus, $t p l$ is a dominant negative mutation for multiple TPL-related proteins [15].

Overexpression of the IAA metabolism-related genes OsGH3.1 and OsCYP71Z2 significantly improved rice resistance to bacterial blight caused by Xanthomonas oryzae pv. oryzae. Therefore, the IAA signaling pathway is implicated in plant defense $[19,20]$. TPL/TPR interact with the transcription complexes involved in auxin signal transduction. Auxins induce the formation of ternary repressor-phytohormone-E3 ligase complexes, which, in turn, cause E3 ligase-catalyzed repressor protein ubiquitination and degradation, and upregulate phytohormone target genes [7]. Auxin-mediated TIR1 (transport inhibitor response 1) E3 ubiquitin ligase binding causes ubiquitination and proteolysis of Aux/IAA repressor protein. At low auxin levels, IAA repressors recruit TPL/TPR to Auxin response factor (ARF) in order to prevent the expression of ARF and its target genes $[21,22]$.

A proteomic analysis showed that AcTPR2 was highly upregulated in kiwifruits following $B$. cinerea infection [23]. Here, we used the 'Hongyang' kiwifruit cultivar as the experimental material. We cloned AcTPR 2 and used the virus-induced gene silencing (VIGS) technique to establish the roles of TPR2 in kiwifruit resistance to $B$. cinerea. We also ran an expression analysis to investigate possible interactions among AcTPR2 and the IAA signaling genes. The results of the present study could help clarify the resistance mechanism responsible for disease resistance in kiwifruit, and furnish genetic resources for molecular breeding of kiwifruit disease resistance.

\section{Results}

\section{Construction of the ACTPR2-TRV2 vector}

A silencing fragment was cloned and its sequence was the same as that of the reference AcTPR2 (Ach25g228601.2-TA, http://kiwifruitgenome.org/). An AcTPR2-TRV2 construct was generated by introducing a 446-bp XbaI/BamHI DNA fragment into a pTRV2 vector (Fig. 1a and b). PCR detection of resistant colonies bearing the AcTPR2-TRV2 construct was performed to confirm that the silencing fragment was successfully ligated onto the pTRV2 vector (Fig. 1c).

\section{AcTPR2 expression was greatly reduced in AcTPR2-TRV fruits}

Agrobacterium GV3101 harboring the AcTPR2-TRV2 expression vector (AcTPR2-TRV), sterilized $\mathrm{ddH}_{2} \mathrm{O}$ (WT), and the vector pTRV1-2 (TRV), were transformed to 'Hongyang' kiwifruit by transient injection. AcTPR2 expression was measured for all three groups. AcTPR2 level was markedly downregulated in the AcTPR2-TRV fruits at 6 days post-injection. In contrast, there were no drastic differences in AcTPR2 expression between the WT and TRV groups (Fig. 2).

\section{Virus-induced silencing of AcTPR2 enhanced kiwifruit susceptibility to $B$. cinerea}

AcTPR2 expression was compared between AcTPR2TRV and control kiwifruit after $B$. cinerea infection. The AcTPR2 level was highest at 4 days post-inoculation (dpi) and the infection time was prolonged in the WT, TRV empty vector, and AcTPR2-TRV fruits. The AcTPR2 levels in these treatments were nearly sixfold higher than they were in the control at $1 \mathrm{dpi}$. However, 


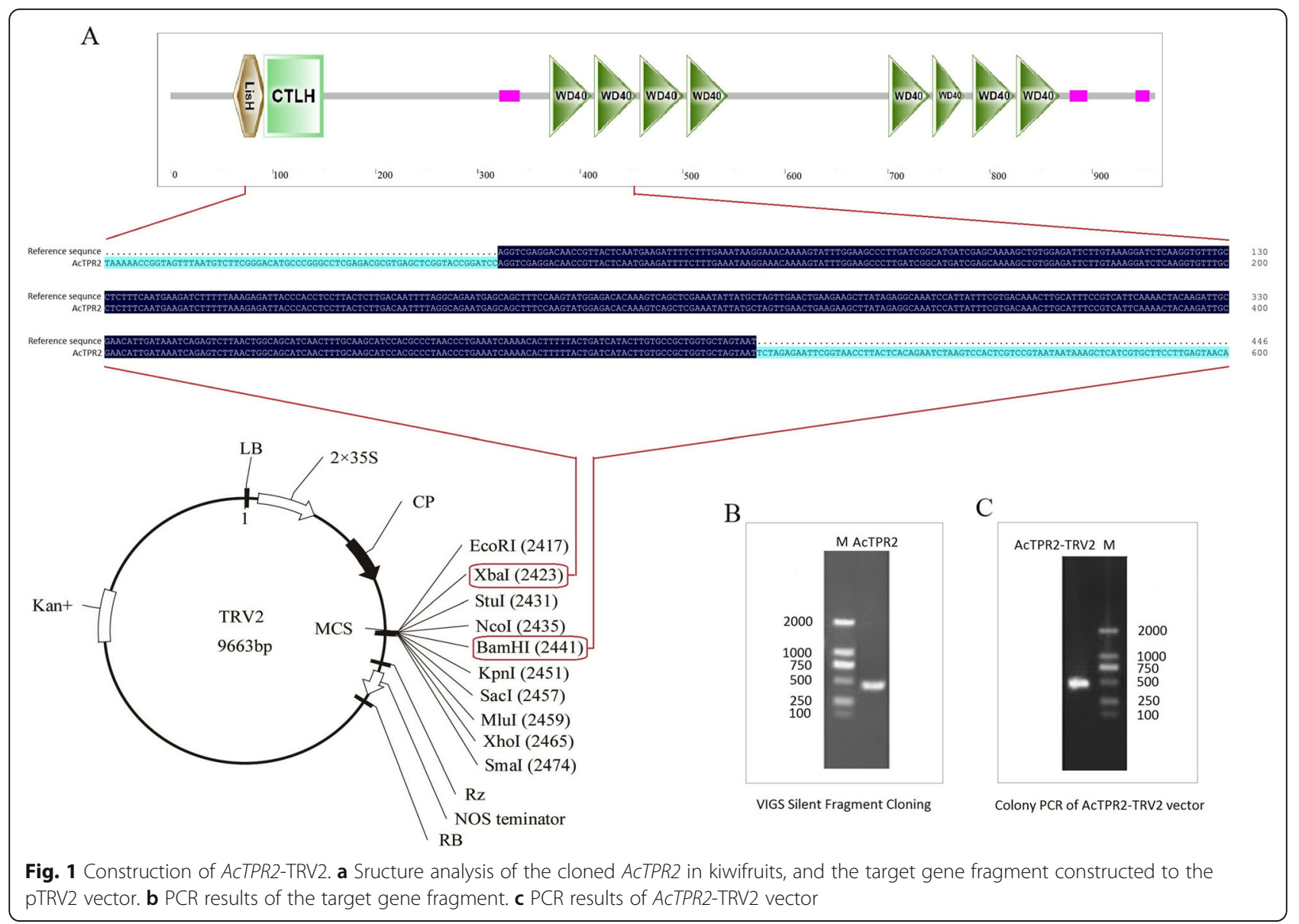

the AcTPR2 level in AcTPR2-TRV was twofold lower than it was at $1 \mathrm{dpi}$ (Fig. 3).

The lesion areas on AcTPR2-silenced kiwifruit were larger than those for the control groups. Furthermore, the injection sites were more susceptible to rot in the AcTPR2-silenced kiwifruit than they were in the control. At $5 \mathrm{dpi}$, the lesion areas on the AcTPR2-TRV fruit were nearly threefold larger than they were on the WT and TRV vector fruits. The $B$. cinerea load was also significantly higher in the AcTPR2-TRV fruits than that in the control at 4 $\mathrm{dpi}$ and $5 \mathrm{dpi}$. Thus, virus-induced silencing of AcTPR2 enhanced kiwifruit susceptibility to $B$. cinerea (Fig. 4).

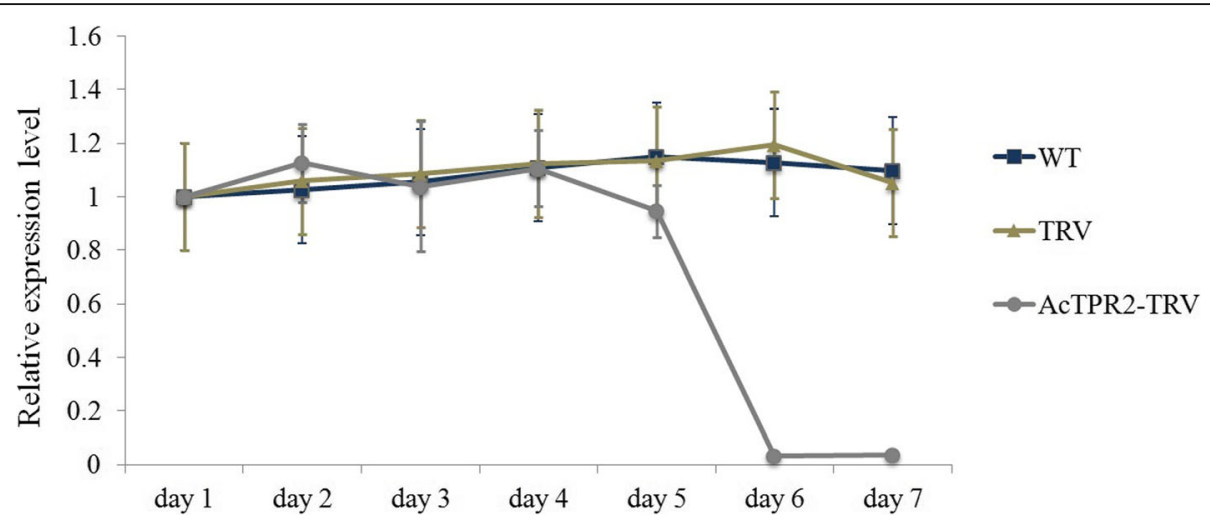

Fig. 2 Relative expression level of AcTPR2 in fruits transformed with $d d H_{2} \mathrm{O}$ water, empty vector and AcTPR2-TRV at 1-7 days. Values are means \pm SE of three biological replicates 


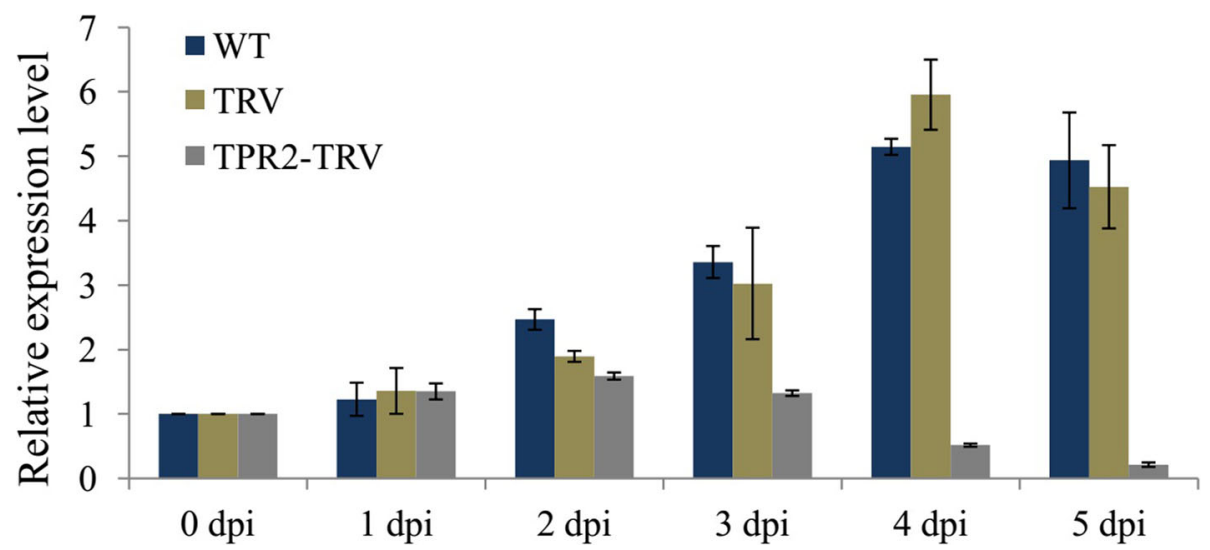

Fig. 3 Relative expression level of AcTPR2 in fruits transformed with $\mathrm{ddH}_{2} \mathrm{O}$ water, empty vector and AcTPR2-TRV upon infection of $B$. cinerea. Values are means \pm SE of three biological replicates

\section{Kiwifruit activate pathogen resistance-related defense enzymes in response to infection}

The activity levels of enzymes such as SOD, POD, CAT, and PAL are indicative of plant disease resistance. These enzymes may be upregulated in response to biotic and abiotic stress and enhance host resistance. The activity levels of all four defense enzymes increased in the control and AcTPR2-TRV kiwifruits in response to $B$. cinerea infection. SOD, POD, and PAL rapidly reacted to pathogenesis at $1 \mathrm{dpi}$ and their activity levels continued to rise until $4 \mathrm{dpi}$, but decreased by $5 \mathrm{dpi}$. CAT was first induced at $2 \mathrm{dpi}$ and its activity steadily increased with infection time. However, at $5 \mathrm{dpi}$, its activity declined. In the absence of $B$. cinerea infection, the activity levels of all four enzymes were much higher in AcTPR2-TRV than they were in the control. Nevertheless, the enzyme activity markedly increased in the AcTPR2-TRV groups under infection stress (Fig. 5).

\section{Increased B. cinerea susceptibility in the AcTPR2-TRV} groups is related to phytohormone interactions TPL/TPR proteins participate in plant signaling pathways and the various phytohormones interact. Here, the relative levels of the phytohormones, IAA, $\mathrm{GA}_{3}$, $\mathrm{ABA}$, and SA, were measured. IAA, GA, and SA levels were observed to sharply increase before $4 \mathrm{dpi}$, and thereafter, they decreased rapidly. In the absence of $B$. cinerea infection, IAA, GA, and SA levels were substantially higher in AcTPR2-TRV than those in the control WT and TRV groups. Upon infection, the levels of IAA, GA, and SA were higher in AcTPR2TRV than they were in the uninfected AcTPR2-TRV at 1-4 dpi. However, the levels of these phytohormones declined at $5 \mathrm{dpi}$ (Fig. 6a, b, and d). In the control, the ABA content continuously increased as the Botrytis cinerea infection prolonged. In case of the AcTPR2-TRV treatment at 1-2 dpi, the ABA content was higher following $B$. cinerea infection than that in the uninfected fruits. After $2 \mathrm{dpi}$, however, the ABA level considerably fell in the AcTPR2-TRV kiwifruit (Fig. 6c).

\section{Virus-induced silencing of ACTPR2-induced IAA signaling gene expression}

Here, we used qRT-PCR to measure the expression levels of the genes governing auxin biosynthesis and signaling in the AcTPR2-TRV and control fruits. AcNIT1 was slightly upregulated in the control fruit within 5 days of storage, but markedly increased upon B. cinerea infection, especially at 1-3 dpi. The AcNIT1 level was higher in the AcTPR2-TRV than that in the control fruits (Fig. 7a). AcARF1and AcARF2 were somewhat upregulated in the control but their levels were fourfold higher in the AcTPR2 silenced fruits than those in the control. $A c A R F 1$ and $A C A R F 2$ were strongly induced in response to $B$. cinerea infection (Fig. $7 \mathrm{~b}$ and c).

\section{Discussion}

TPL/TPR expression may be associated with plantpathogen interactions [24]. To the best of our knowledge, however, no prior study has explored the roles of TPL/TPR in kiwifruit- $B$. cinerea interaction. Previous research showed that AcTPR2 is highly expressed in kiwifruit following B. cinerea infection [23], and therefore, it can potentially play a crucial role in this process. Here, we applied expression profiling, transgenic studies, and infection analysis to investigate the functions of AcTPR2 in disease resistance in kiwifruit- $B$. cinerea interactions.

We used the VIGS method to examine the reverse function of AcTPR2 in kiwifruit resistance to B. cinerea. A qRT-PCR analysis showed that AcTPR2 was downregulated in AcTPR2-TRV2 kiwifruits 6 days after transformation and the transformed vectors had a silencing 


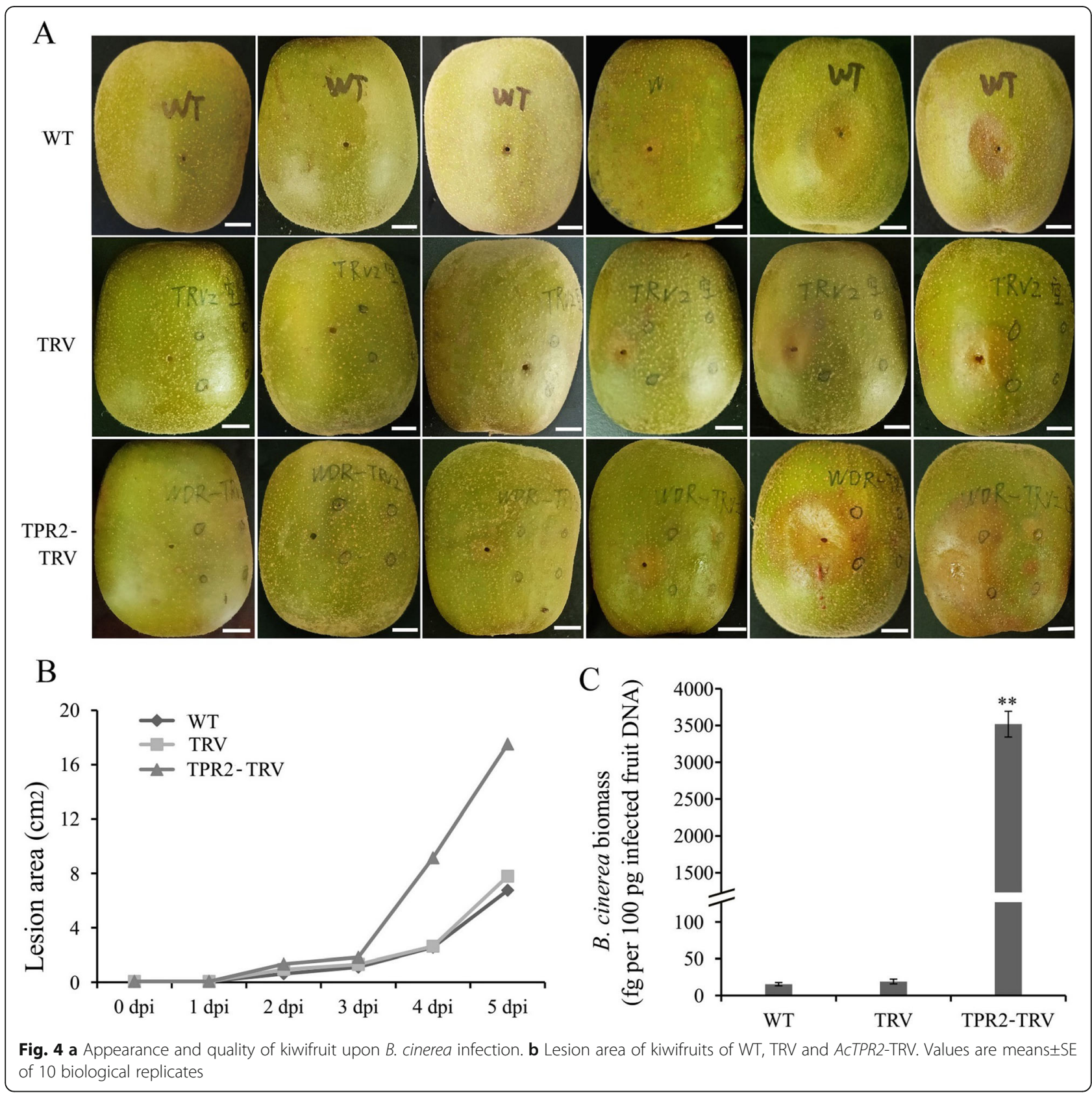

effect (Fig. 2). AcTPR2 was markedly upregulated in the kiwifruits after $B$. cinerea infection. Hence, AcTPR2 may have a defensive function against $B$. cinerea in kiwifruit (Fig. 3). However, AcTPR2 expression was substantially lower in the AcTPR2-TRV2 kiwifruits than that in the controls. The foregoing results validated the silencing effect identified by VIGS.

TPR2 overexpression in plants might enhance pathogen resistance. Nevertheless, little research has been conducted on the effects of TPR2 downregulation [24]. Here, we provided reverse evidence for the role for TPR2 in fungal pathogen resistance in kiwifruit. Virus- induced silencing of AcTPR2 increased B. cinerea susceptibility in kiwifruit. These findings were consistent with a previous study reporting that AtTPR1 overexpression activated Arabidopsis defense responses, even though various TPRs were redundant [24].

Activation of a defense response is accompanied by the induction of pathogenesis-related enzymes. Stress induces excessive generation of reactive oxygen species (ROS). SOD, POD, and CAT are vital plant antioxidant enzymes that remove active oxygen produced in response to external damage [25]. PAL is a critical enzyme in the phenylpropane metabolic pathway, which 


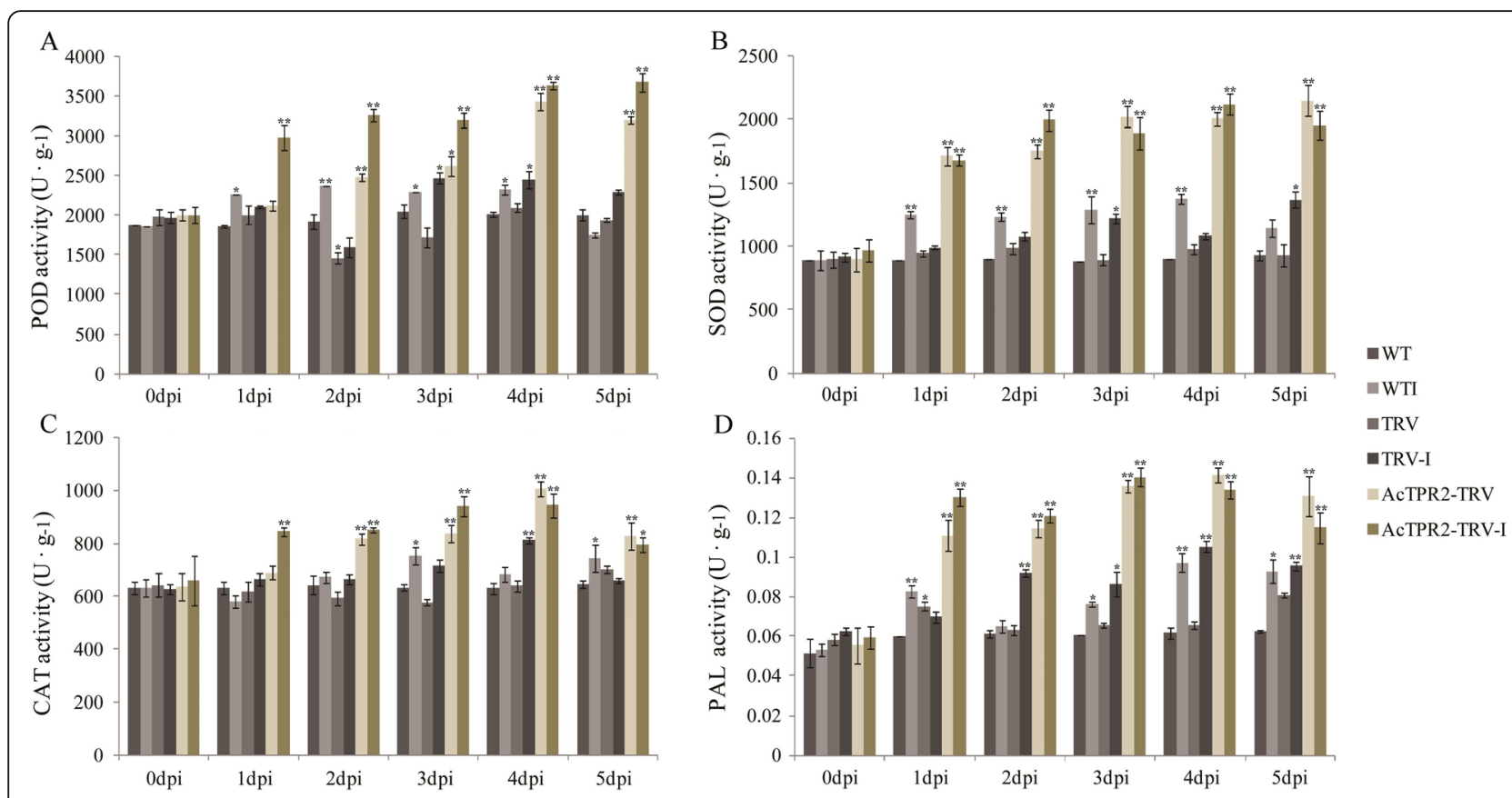

Fig. 5 Effects of $B$. cinerea infection on activities of 4 defense enzymes in kiwifruit of WT, TRV and ACTPR2-TRV. The activity of antioxidant enzymes including SOD, POD, CAT and PAL were detected. Values are means \pm SE of three biological replicates. " "*" represent the significant difference $(p<$ 0.05), while "**" represent the extremely significant difference $(p<0.01)$

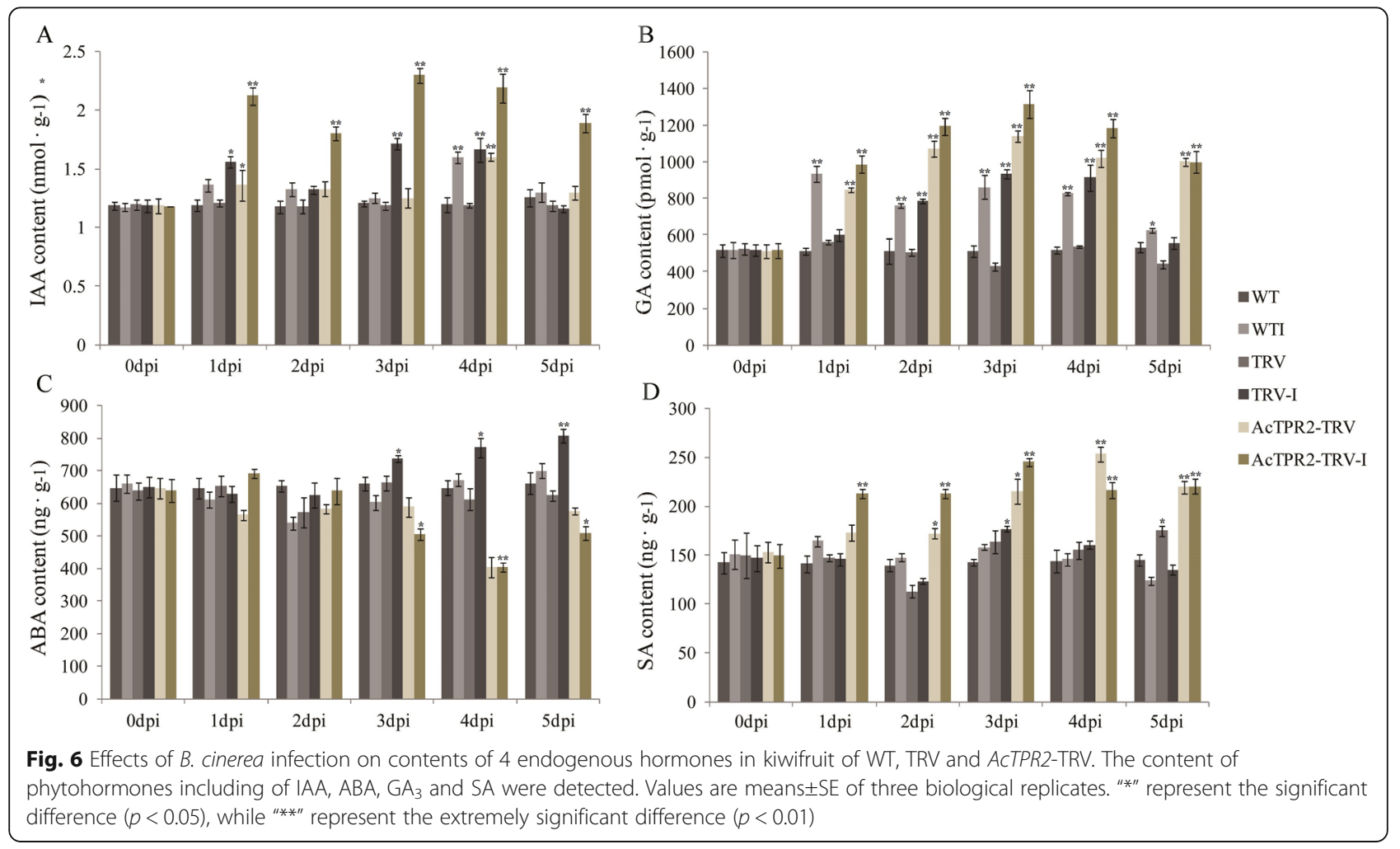



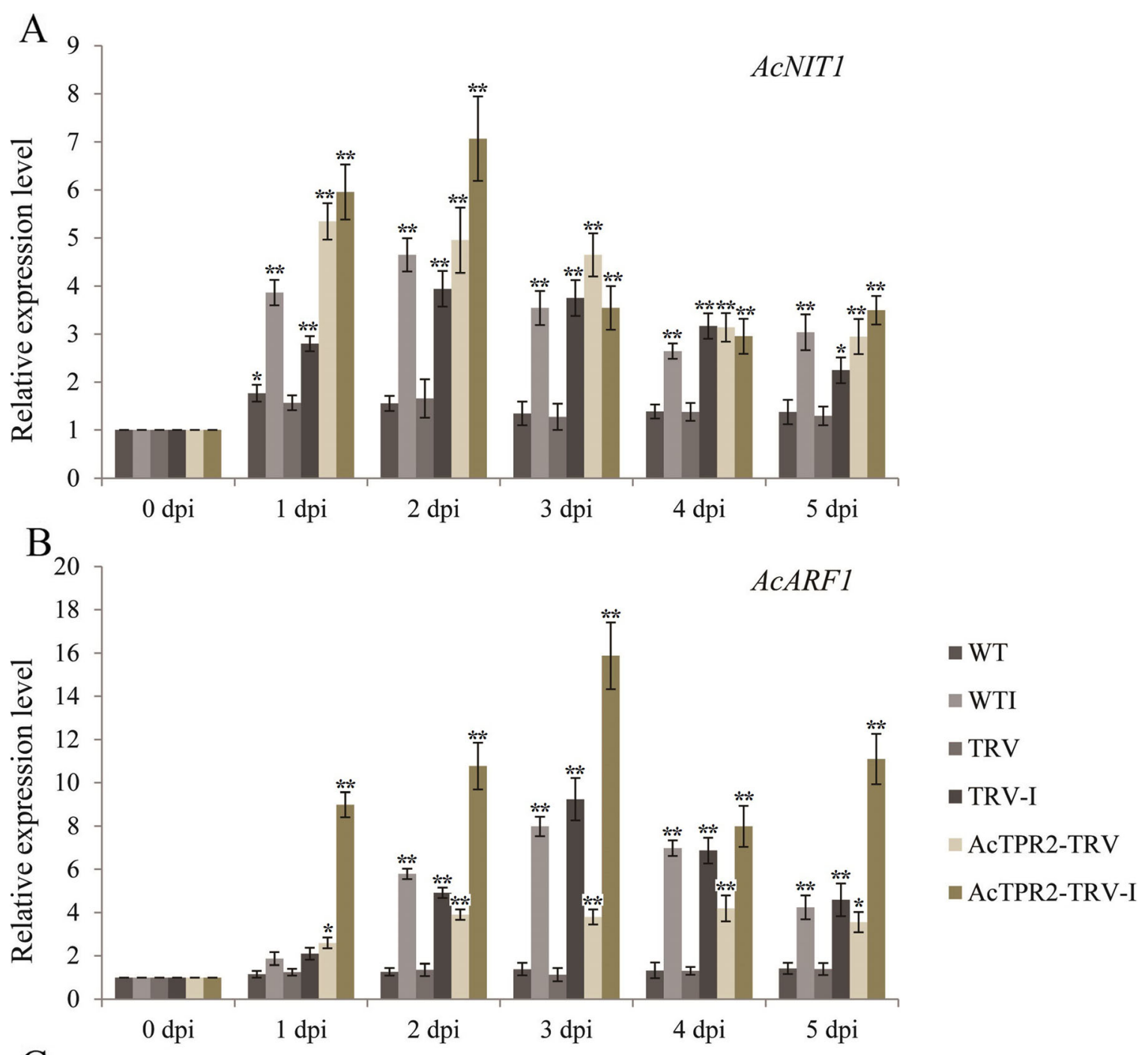

C

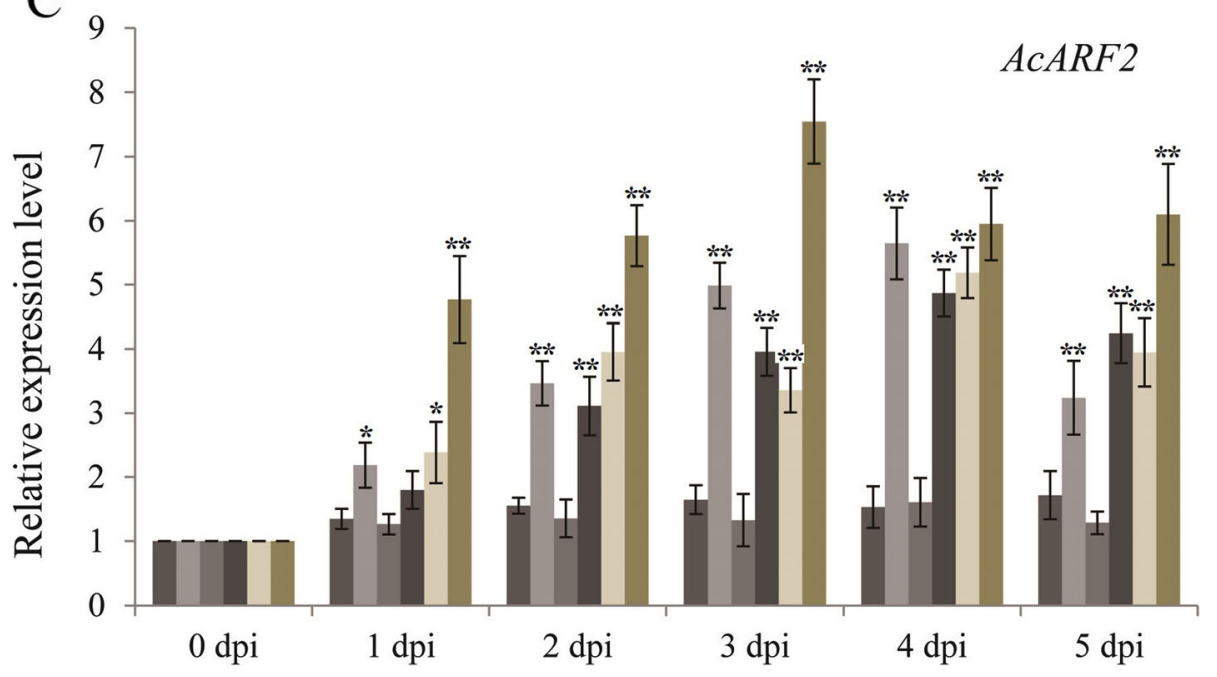

Fig. 7 Expression analysis of IAA signaling genes in kiwifruits of WT, TRV and ACTPR2-TRV upon B. cinerea infection. Values are means \pm SE of three biological replicates. "**" represent the significant difference $(p<0.05)$, while ${ }^{~} * * *$ represent the extremely significant difference $(p<0.01)$ 
generates various secondary metabolites and prevents pathogen invasion [26]. Here, all four defense enzymes were upregulated in the control and the AcTPR2-TRV2 kiwifruits in the presence of Botrytis cinerea. The activity levels of SOD, POD, CAT, and PAL increased in response to the pathogen infection until $4 \mathrm{dpi}$ and decreased thereafter. The onset of infection at $4 \mathrm{dpi}$ might have indicated a breakdown in localized plant defense responses (Fig. 5). In the absence of infection, all four enzymes were markedly upregulated in AcTPR2TRV2 as compared to those in the control. Under $B$. cinerea infection stress, however, the activity levels of all four enzymes drastically increased in the AcTPR2-TRV2 treatment (Fig. 5). Thus, after the AcTPR2 gene was silenced, the kiwifruit internally upregulated these enzymes. Nevertheless, the AcTPR2-TRV2 kiwifruits were relatively more susceptible to rot.

TPL/TPR interact with the transcription complexes involved in the phytohormone pathways, especially in auxin signal transduction [7, 14]. Various phytohormones coordinate plant growth and stress adaptation. Here, in the absence of Botrytis cinerea infection, the IAA, GA, and SA levels were considerably higher in AcPGIP-TRV2 than those in the control. Therefore, AcPGIP silencing enables kiwifruit to activate phytohormone signaling pathways promptly via other mechanisms and defend itself against $B$. cinerea. This finding was consistent with that of a previous study stating that plants produce endogenous phytohormones that adjust the pathogen response [20]. The levels of all endogenous phytohormones in AcPGIP-TRV2 increased but then decreased to lower levels than those of the control. Further, there were synergies among IAA, GA, and SA. The
ABA content was higher in the fruit at 1-2 dpi than that in the uninfected fruit. However, the ABA level dramatically decreased after 2 dpi. This pattern was not observed for the other three phytohormones.

In this study, we evaluated the expression levels of three IAA signaling genes. Prolonged storage slightly increased AcNIT1 in the control fruit. Thus, IAA participated in fruit ripening and senescence. Botrytis cinerea infection accelerated fruit senescence by inducing host IAA biosynthesis and promoting expression of IAA signaling genes such as AcNIT1, AcARF1, and AcARF2. Moreover, the expression levels of all three genes were higher in AcTPR2-TRV than those in the control. There were relatively elevated IAA levels and a more severe rot phenotype in the AcTPR2-TRV kiwifruit. Hence, AcTPR2 downregulation might promote the expression of IAA and IAA signaling genes and accelerate postharvest kiwifruit senescence. Further, Botrytis cinerea infection drastically increased the relative AcTPR 2 responses. We, therefore, propose that AcTPR2 enhances plant pathogen defense by downregulating IAA and IAA signaling genes (Fig. 8). The results of the present study are consistent with those of a previous research reporting that Arabidopsis overexpressing AtTRP1 showed a comparatively weak response to exogenous IAA and demonstrated altered expression of a subset of auxin early response genes [27].

\section{Conclusion}

The virus-induced gene silencing technique was used to establish the functions of the AcTPR2 gene in kiwifruit resistance to Botrytis cinerea. Virus-induced silencing of $A c T P R 2$ increased kiwifruit sensitivity to $B$. cinereal

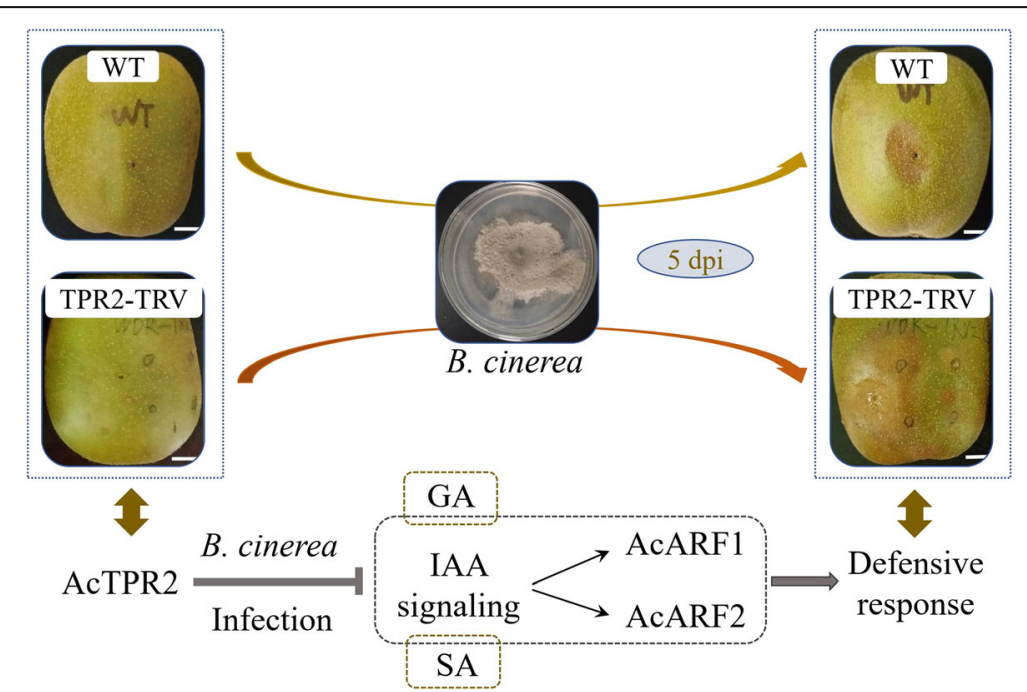

Fig. 8 A proposed model for the present study. ACTPR2 could enhance the defensive ability of kiwifruits against B. cinerea by negatively regulate IAA and IAA signaling genes. While GA and SA have synergistic effect with IAA, and jointly respond to pathogen infection 
infestations. The antioxidant enzymes SOD, POD, CAT, and PAL and the endogenous phytohormones IAA, GA, $\mathrm{ABA}$, and SA were all activated in response to pathogeninduced stress and injury in kiwifruit. The IAA signaling genes AcNIT, AcARF1, and AcARF2 were all upregulated in kiwifruit transformed by AcTPR2-TRV compared to the control. Since IAA levels were also higher and the gray mold rot phenotype was more intense in AcTPR2TRV kiwifruits than they were in the control, AcTPR2 downregulation promotes the IAA and IAA signaling genes and accelerates senescence in postharvest kiwifruit. Botrytis cinerea substantially upregulated AcTPR2 in the kiwifruit compared with the control. Thus, AcTPR2 augments kiwifruit defense against pathogens by downregulating the IAA and IAA signaling genes.

\section{Methods}

\section{Plant materials}

Kiwifruit (Actinidia chinensis cv. Hongyang) is a fruit crop of high economic importance and has interested consumers [28]. The variety passed the approval of the Sichuan Provincial Crop Variety Approval Committee and was named Kiwifruit 'Hongyang' in 1977. Jianmin Tang undertook the formal identification of the plant material used in the present study [3]. Nearly mature, undamaged, and pest-free kiwifruit were harvested at 130 days after flowering in an experimental facility in Kaizhou District, Chongqing, China $\left(31^{\circ} 23^{\prime} \mathrm{N}, 108^{\circ} 39^{\prime}\right.$ E). Kiwifruit with an average weight of $95 \mathrm{~g}$ were transported to the laboratory within $5 \mathrm{~h}$ of harvest. Fruits were further disinfected with $2 \%(\mathrm{v} / \mathrm{v})$ sodium hypochlorite for $2 \mathrm{~min}$, rinsed with tap water, and air-dried.

\section{Vector constructions and transformation}

Total RNA was extracted from the kiwifruit using RNAiso Plus reagents (Takara Biomedical Technology Co. Ltd., Beijing, China) and reverse-transcribed into cDNA with a PrimeScript ${ }^{\mathrm{Tm}}$ RT Reagent Kit (Takara Biomedical Technology Co. Ltd., Beijing, China). The single electrophoretic band of the AcTPR2 PCR product was recovered and cloned into a $\mathrm{pMD}^{\circ} 19-\mathrm{T}$ Simple Vector (Takara Biomedical Technology Co. Ltd., Beijing, China). PCR-positive bacterial colonies was sequenced to verify AcTPR2 correctness. An AcTPR2-TRV2 construct was generated by introducing a 446-bp XbaI/BamHI DNA fragment into a pTRV2 vector (Fig. 1). The AcTPR2 gene fragment and the PTRV2 vector were double-digested by $\mathrm{Xba \textrm {I } /}$ $B a m \mathrm{HI}$, recovered, ligated, transformed into E. coli DH $5 \alpha$-competent cells, and incubated on a resistant plate medium containing $50 \mathrm{mg} \mathrm{L}^{-1}$ kanamycin. Resistant colonies were detected by PCR and the PCRpositive bacterial colonies were selected to extract plasmids. The universal vector primer RV-XIAYOU
(5-'AACCTAAAACTTCAGACACG-3') was used for sequencing. The AcTPR2-TRV2 sequence was the same as the reference sequence (Fig. 1). The AcTPR2TRV2 expression vector was then introduced into Agrobacterium tumefaciens strain GV3101. Transformed A. tumefaciens harboring the pTRV2 vector was mixed in a 1:1 ratio with $A$. tumefaciens GV3101 bearing the pTRV1 vector. The mixed Agrobacterium cultures with density $(\mathrm{OD} 600=1.0)$ were injected by syringe into kiwifruit. Sterilized $\mathrm{ddH}_{2} \mathrm{O}$ and vector TRV (pTRV1:pTRV2 = 1:1) served as the control.

\section{Infection by $B$. cinerea}

Strain HFXC-16 of $B$. cinerea isolated from infected kiwifruit was incubated at $25^{\circ} \mathrm{C}$ and grown on potato dextrose agar (PDA) for 2 weeks [29] (Chen et al., 2015). Seven days after injection of the fruit with Agrobacterium tumefaciens, sterilized $\mathrm{ddH}_{2} \mathrm{O}$, or TRV1-2 vector, $10 \mu \mathrm{L}$ B. cinerea spore suspension containing $10^{4}$ spores $\mathrm{mL}^{-1}$ was injected by syringe into each wound induced near those formed by previous Agrobacterium or $\mathrm{ddH}_{2} \mathrm{O}$ injections. The treated kiwifruit were placed in covered plastic food trays, enclosed in polyethylene bags, and stored at $25^{\circ} \mathrm{C}$ in a constant-temperature incubator (Sanyo Electric Co. Ltd., Osaka, Japan).

\section{B. cinerea DNA quantification}

Genomic DNA was isolated from samples of $B$. cinerea-infected kiwifruit at $5 \mathrm{dpi}$. The genomic DNA of $B$. cinerea was tested using the universal primers ITS1/ITS4 and a 551-bp band was obtained. Fifty picograms DNA was subjected to qRT-PCR analysis of the ITS sequence using primers designed from the sequenced 551-bp fragment (Table 1).

\section{qRT-PCR for expression analysis}

Kiwifruit tissues were collected daily for 6 days. The experimental design consisted of three replicates of 10 fruits per treatment and the experiment was repeated thrice. All tissues were stored at $-80{ }^{\circ} \mathrm{C}$ until RNA extraction. The total RNA was purified with RNAiso Plus Kits (TaKaRa Bio Inc., Shiga, Japan) following the manufacturer's protocol. One microgram RNA was treated with RNase-free DNase I (Thermo Fisher Scientific, Waltham, MA, USA) to eliminate genomic DNA and reverse-transcribed with oligo $(\mathrm{dT})$ using a TransScript All-in-One First-Strand cDNA Synthesis Supermix for qPCR (One-Step gDNA Removal) Kit (TransGen Biotech Co. Ltd., Beijing, China). A TB Green $^{\text {nm }}$ Premix Ex Taq ${ }^{\text {mix }}$ (Tli RNaseH Plus; TaKaRa Bio Inc., Shiga, Japan) kit was used for qRT-PCR analysis. The $\beta$-actin gene served as an internal control [23]. 
Table 1 Primers used for PCR and qRT-PCR

\begin{tabular}{|c|c|c|c|}
\hline Purpose & Name & Sequence $\left(5^{\prime}-3^{\prime}\right)$ & \\
\hline \multirow[t]{2}{*}{ AcTPR2-pTRV2 construction } & AcTPR2 & $\mathrm{F}$ & (Xbal)GCTCTAGAATTACTAGCACCAGCGGCAC \\
\hline & & $\mathrm{R}$ & (BamHI)CGGGATCCAGGTCGAGGACAACCGTTAC \\
\hline \multirow[t]{2}{*}{ PCR } & ITS & $\mathrm{F}$ & TCCGTAGGTGAACCTGCGC \\
\hline & & $\mathrm{R}$ & TCCTCCGCTTATTGATATGC \\
\hline \multirow[t]{2}{*}{ qRT-PCR } & ITS & $\mathrm{F}$ & CTGTTCGAGCGTCATTTCAA \\
\hline & & $\mathrm{R}$ & CCTACCTGATCCGAGGTCAA \\
\hline \multirow[t]{2}{*}{ qRT-PCR } & Actin & $\mathrm{F}$ & GCAGTGTTTCCCAGTATTGT \\
\hline & & $\mathrm{R}$ & TCCATGTCATCCCAGTTGC \\
\hline \multirow[t]{2}{*}{ qRT-PCR } & ACTPR2 & $\mathrm{F}$ & GGGCTGCGTATACTGAGGAT \\
\hline & & $\mathrm{R}$ & TTGCTTGCTGTCAAAGGGTC \\
\hline \multirow[t]{2}{*}{ qRT-PCR } & ACNIT1 & $\mathrm{F}$ & TTGTITGTCAGCCAACCA \\
\hline & & $\mathrm{R}$ & ATGCCCAACCACATCAAAAT \\
\hline \multirow[t]{2}{*}{ qRT-PCR } & ACARF1 & $\mathrm{F}$ & TTGTGTGCCATTAGGCATGT \\
\hline & & $\mathrm{R}$ & TGGTTTCCACTTGGTTAGGC \\
\hline \multirow[t]{2}{*}{ qRT-PCR } & ACARF2 & $\mathrm{F}$ & AGGGTCTTGCAAAGTCGAGA \\
\hline & & $\mathrm{R}$ & GATCGAGTCCCAAAACCTGA \\
\hline
\end{tabular}

Primers are listed in Table 1. The PCR products were cloned and sequenced to verify their identity.

\section{Analysis of defensive enzymes}

One half gram kiwifruit tissue was extracted in $10 \mathrm{~mL}$ of $100 \mathrm{mM}$ potassium phosphate buffer ( $\mathrm{pH}$ 6.8) and centrifuged at $12,000 \times g$ and $4{ }^{\circ} \mathrm{C}$ for $20 \mathrm{~min}$. The supernatant was collected for enzyme extraction and determination. The nitrogen blue tetrazole (NBT) photoreduction method was used to determine SOD activity [30]. The enzyme dosage preventing 50\% NBT photochemical reduction was treated as $1 \mathrm{Ug}^{-1}$. POD activity was estimated by the guaiacol method [31]. CAT activity was evaluated by the ultraviolet absorption method and defined as $1 \mathrm{U}$ enzyme activity when the optical density (OD) at $240 \mathrm{~nm} \mathrm{~min}^{-1}$ (A240) was reduced by $0.1\left(\mathrm{Ug}^{-1}\right)$. PAL activity was determined by a previously reported method [32]. The fruit samples were extracted in $50 \mathrm{mM}$ Tris buffer (pH 8.5) and centrifuged at $6000 \times g$ and $4{ }^{\circ} \mathrm{C}$ for $10 \mathrm{~min}$ to collect the supernatant. $L$-phenylalanine and $100 \mu \mathrm{L}$ of 2 $\mathrm{N} \mathrm{HCl}$ were added to the supernatant to initiate the reaction. Absorbance was recorded at $290 \mathrm{~nm}$ and indicated cinnamic acid formation.

\section{Liquid chromatograph-mass spectrometer (LC-MS) for phytohormone analysis}

To determine the IAA, $\mathrm{GA}_{3}$, and ABA content, $3 \mathrm{~g}$ kiwifruit tissue was weighed, frozen, powdered in liquid nitrogen, and later analyzed by LC-MS as previously described [33]. Each treatment group was represented by three independent biological replicates.

\section{Statistical analysis}

Statistical analyses were performed with the software package, SPSS 18.0. The student $\mathrm{T}$ test was used to compare defensive enzyme, phytohormone content or relative gene expression in the control and various infection groups. Statistical significance was defined as $p<0.05$.

\section{Supplementary Information}

The online version contains supplementary material available at https://doi. org/10.1186/s12870-020-02773-x.

\section{Additional file 1.}

Additional file 2.

\section{Abbreviations}

ABA: Abscisic acid; ARF: Auxin response factor; CAT: Catalase; CTLH: Cterminal to the LisH; dpi: Days post-inoculation; EAR: Ethylene-responsive element binding factor-associated amphiphilic repression; $G_{3}$ : Gibberellic acid; IAA: Indole-3-acetic acid; LC-MS: Liquid chromatography-mass spectrometry; LisH: Lissencephaly homologous; NBT: Nitro blue tetrazole; OD: Optical density; PAL: Phenylalanine ammonia-lyase; PDA: Potato dextrose agar; POD: Peroxidase; qRT-PCR: Quantitative reverse-transcription polymerase chain reaction; ROS: Reactive oxygen species; SA: Salicylic acid;

SOD: Superoxide dismutase; TIR: Transport inhibitor response; TPD: TOPLESS domain; TPL: TOPLESS; TPR: TOPLESS-RELATED; VIGS: Virus-induced gene silencing; WUS: WUSCHEL

\section{Acknowledgements}

Not applicable.

\section{Authors' contributions}

ZL conceived, designed and carried out the study and wrote the manuscript. $J \mathrm{~L}, \mathrm{QL}$ and JT provided important suggestions on the experimental design and analyses. YL offered some help during the experimental operation. QL and JT helped to modify the manuscript. All authors read and approved the manuscript. 


\section{Funding}

The authors are grateful for the financial support provided by National Natural Science Foundation of China (32001351 and 31670688) to conduct research design and most of the experiment, Natural Science Foundation of Chongqing (cstc2018jscx-msybX0196) in data analysis and manuscript writing, and to cultivate materials used in the present study.

\section{Availability of data and materials}

The kiwifruit genome database can be found in http://kiwifruitgenome.org/. The plant materials are available from the corresponding author on request.

\section{Ethics approval and consent to participate}

Not applicable.

\section{Consent for publication}

Not applicable.

\section{Competing interests}

The authors declare that they have no competing interest.

Received: 22 July 2020 Accepted: 2 December 2020

Published online: 10 December 2020

\section{References}

1. Park YS, Im MH, Gorinstein S. Shelf life extension and antioxidant activity of 'Hayward' kiwi fruit as a result of prestorage conditioning and 1methylcyclopropene treatment. J Food Sci Technol. 2015;52:2711-20.

2. Bardas GA, Veloukas T, Koutita O, Karaoglanidis GS. Multiple resistance of Botrytis cinerea from kiwifruit to SDHIs, Qols and fungicides of other chemical groups. Pest Manag Sci. 2010;66:967-73.

3. Tang J, Liu Y, Li H, Wang L, Huang K, Chen Z. Combining an antagonistic yeast with harpin treatment to control postharvest decay of kiwifruit. Biol Control. 2015:89:61-7.

4. Hua C, Kai K, Wanling Bi W, Shi W, Liu Y, Zhang D. Curcumin induces oxidative stress in Botrytis cinerea, resulting in a reduction in gray mold decay in kiwifruit. J Agric Food Chem. 2019;67:7968-76.

5. Luo A, Bai J, Liu R, Fang Y. Effects of ozone treatment on the quality of kiwifruit during postharvest storage affected by Botrytis cinerea and Penicillium expansum. J Phytopathol. 2019;167:470-8.

6. Kieffer M, Stern Y, Cook H, Clerici E, Maulbetsch C, Laux T, Davies B. Analysis of the transcription factor WUSCHEL and its functional homologue in Antirrhinum reveals a potential mechanism for their roles in meristem maintenance. Plant Cell. 2006;18:560-73.

7. Ke J, Ma H, Gu X, Thelen A, Brunzelle JS, Li J, Xu HE, Melcher K. Structural basis for recognition of diverse transcriptional repressors by the TOPLESS family of corepressors. Sci Adv. 2015;1:e1500107.

8. Collins J, O'Grady K, Chen S, Gurley W. The C-terminal WD40 repeats on the TOPLESS co-repressor function as a protein-protein interaction surface. Plant Mol Biol. 2019;100:47-58.

9. Martin-Arevalillo R, Nanao MH, Larrieu A, Vinos-Poyo T, Mast D, GalvanAmpudia C, Brunoud G, Vernoux T, Dumas R, Parcy F. Structure of the Arabidopsis TOPLESS corepressor provides insight into the evolution of transcriptional repression. Proc Natl Acad Sci U S A. 2017;114:8107-12.

10. Braun P, Carvunis A-R, Charloteaux B, Dreze M, Ecker JR, Hill DE, Roth FP, Vidal M, Galli M, Balumuri P, et al. Evidence for network evolution in an Arabidopsis interactome map. Science. 2011;333:601-7.

11. Causier B, Ashworth M, Guo W, Davies B. The TOPLESS interactome: a framework for gene repression in Arabidopsis. Plant Physiol. 2012;158: 423-38.

12. Shyu C, Figueroa P, Depew CL, Cooke TF, Sheard LB, Moreno J, Katsir L, Zheng N, Browse J, Howe GA. JAZ8 lacks a canonical degron and has an EAR motif that mediates transcriptional repression of jasmonate responses in Arabidopsis. Plant Cell. 2012;24:536-50.

13. Fukazawa J, Teramura H, Murakoshi S, Nasuno K, Nishida N, Ito T, Yoshida M, Kamiya Y, Yamaguchi S, Takahashi Y. DELLAs function as coactivators of GAlASSOCIATED Factor1 in regulation of gibberellin homeostasis and signaling in Arabidopsis. Plant Cell. 2014;26:2920-38.

14. Lee M-S, An J-H, Cho HT. Biological and molecular functions of two EAR motifs of Arabidopsis IAA7. J Plant Biol. 2016;59:24-32.

15. Long J, Ohno C, Smith ZR, Meyerowitz EM. TOPLESS regulates apical embryonic fate in Arabidopsis. Science. 2006;312:1520-3.
16. Jiang L, Liu X, Xiong G, Liu H, Chen F, Wang L, Meng X, Liu G, Yu H, Yuan Y, et al. DWARF 53 acts as a repressor of strigolactone signalling in rice. Nature. 2013;504:401-5.

17. Ryu $\mathrm{H}$, Cho H, Bae W, Hwang I. Control of early seedling development by BES1/TPL/HDA19-mediated epigenetic regulation of ABI3. Nat Commun. 2014;5:4138.

18. Goralogia GS, Liu T-K, Zhao L, Panipinto PM, Groover ED, Bains YS, Imaizumi T. CYCLING DOF FACTOR 1 represses transcription through the TOPLESS COrepressor to control photoperiodic flowering in Arabidopsis. Plant J Cell Mol Biol. 2017;92:244-62.

19. Fu J, Liu H, Li Y, Yu H, Li Z, Xiao J, Wang S. Manipulating broad-spectrum disease resistance by suppressing pathogen-induced auxin accumulation in rice. Plant Physiol. 2011;155:589-602.

20. Li W, Wang F, Wang J, Fan F, Zhu J, Yang J, Liu F, Zhong W. Overexpressing CYP71Z2 enhances resistance to bacterial blight by suppressing auxin biosynthesis in rice. PLoS One. 2015;10:e0119867.

21. Peer WA. From perception to attenuation: Auxin signalling and responses. Curr Opin Plant Biol. 2013;16:561-8.

22. Wang R, Estelle M. Diversity and specificity: Auxin perception and signaling through the TIR1/AFB pathway. Curr Opin Plant Biol. 2014;21:51-8.

23. Liu J, Sui Y, Chen H, Liu Y, Liu Y. Proteomic analysis of kiwifruit in response to the postharvest pathogen, Botrytis cinerea. Front Plant Sci. 2018;9:158.

24. Zhu Z, Xu F, Zhang Y, Cheng YT, Wiermer M, Li X, Zhang Y. Arabidopsis resistance protein $\mathrm{SNC} 1$ activates immune responses through association with a transcriptional corepressor. Proc Natl Acad Sci U S A. 2010;107: 13960-5.

25. El-Esawi MA, Alayafi AA. Overexpression of StDREB2 transcription factor enhances drought stress tolerance in cotton (Gossypium barbadense L.). Genes. 2019;10:142.

26. Khademi Astane R, Bolandnazar S, Zaare Nahandi F, Oustan S. Effect of selenium application on phenylalanine ammonia-lyase (PAL) activity, phenol leakage and total phenolic content in garlic (Allium sativum L.) under $\mathrm{NaCl}$ stress. Inform Proc Agric. 2018;5:339-44.

27. Lin Z, Ho C-W, Grierson D. AtTRP1 encodes a novel TPR protein that interacts with the ethylene receptor ERS1 and modulates development in Arabidopsis. J Exp Bot. 2009;60:3697-714.

28. Lin M, Fang J, Qi X, Li Y, Chen J, Sun L, Zhong Y. iTRAQ-based quantitative proteomic analysis reveals alterations in the metabolism of Actinidia arguta. Sci Rep. 2017;7:5670.

29. Chen H, Cheng Z, Wisniewski M, Liu Y, Liu J. Ecofriendly hot water treatment reduces postharvest decay and elicits defense response in kiwifruit. Environ Sci Pollut Res Int. 2015;22:15037-45.

30. Ries CNGA. Superoxide Dismutases: I. Occurrence in Higher Plants. Plant Physiol. 1977;59:309-14.

31. Chance B, Maehly AC. Assay of catalase and peroxidases. Methods Enzymol. 1955;2:764-75.

32. Beaudoin-Eagan LD, Thorpe TA. Tyrosine and phenylalanine Ammonia Lyase activities during shoot initiation in tobacco callus cultures. Plant Physiol. 1985;78:438-41.

33. Gou J, Strauss SH, Tsai CJ, Fang K, Chen Y, Busov JX, V.B. Gibberellins regulate lateral root formation in Populus through interactions with Auxin and other hormones. Plant Cell. 2010;22:623-39.

\section{Publisher's Note}

Springer Nature remains neutral with regard to jurisdictional claims in published maps and institutional affiliations.

Ready to submit your research? Choose BMC and benefit from:

- fast, convenient online submission

- thorough peer review by experienced researchers in your field

- rapid publication on acceptance

- support for research data, including large and complex data types

- gold Open Access which fosters wider collaboration and increased citations

- maximum visibility for your research: over $100 \mathrm{M}$ website views per year

At BMC, research is always in progress.

Learn more biomedcentral.com/submission 